\title{
Representaciones sobre las lenguas de un grupo de estudiantes indígenas en un programa de formación de docentes de idiomas ${ }^{1}$
}

\section{Language Representations of a group of Indigenous Students in a Language Teacher Education Program}

\author{
Fabio Alberto Arismendi² \\ Denis Ramirez ${ }^{3}$ \\ Sara Arias ${ }^{4}$
}

Citation/ Para citar este Artículo: Arismendi F., Ramirez D. \& Arias S. (2016). Representaciones sobre las lenguas de un grupo de estudiantes indígenas en un programa de formación de docentes de idiomas. Colomb.Appl.Linguist.J. 18(1), pp 84-97

Received: 06-May-2015 / Accepted: 15-Feb-2016

DOI: http://dx.doi.org/10.14483/calj.v18n1.8598

\section{Resumen}

Los procesos de aprendizaje de lenguas extranjeras de estudiantes provenientes de comunidades indígenas han sido poco explorados en Colombia. En este artículo se presentan hallazgos parciales de una investigación que busca explorar los retos que los estudiantes de pueblos ancestrales enfrentan al estudiar lenguas en una universidad pública. Partiendo de la teoría de las representaciones sociales, nos enfocamos en este texto en las representaciones que los estudiantes participantes tienen tanto de sus lenguas maternas como de las lenguas objeto de estudio, inglés y francés. Con este fin, realizamos un estudio de caso cualitativo en el cual participaron ocho estudiantes indígenas. Los datos recogidos por medio de entrevistas individuales y colectivas, observaciones de clase y grupos focales nos permitieron conocer los repertorios lingüísticos de los participantes, así como las representaciones de sus lenguas maternas y extranjeras. Los hallazgos muestran que los profesores juegan un rol importante en la construcción de las representaciones y que estas a su vez evolucionan a lo largo del proceso de aprendizaje.

Palabras claves: competencia plurilingüe y pluricultural, lengua extranjera, lengua materna.

\begin{abstract}
Few studies have explored the foreign language learning processes of indigenous students in Colombia. In this article, we present preliminary findings of a research project which aims to explore the challenges that indigenous students face when learning languages in a public university. Based on the theory of social representations, we focus in this text on the representations that participant students have regarding their mother tongue as well as the languages

1 Este artículo reporta hallazgos parciales del proyecto de investigación "Retos académicos, lingüísticos y sociales en el aprendizaje del inglés y el francés de los estudiantes indígenas de un programa de licenciatura en lenguas extranjeras de una universidad pública", financiado por la Escuela de Idiomas de la Universidad de Antioquia y realizado entre febrero de 2014 y febrero de 2016.

2 Universidad de Antioquía. Antioquía. Colombia. fabio.arismendi@udea.edu.co

3 Universidad de Antioquía. Antioquía. Colombia. deniseramjim@yahoo.fr

4 Universidad de Antioquía. Antioquía. Colombia. sarariasp@outlook.com
\end{abstract}


they study, English and French. We carried out a qualitative case-study in which eight students from different ancestral communities participated. Data gathered from individual and collective interviews, class observations and focus groups allowed us to uncover the linguistic repertoires of participants as well as the representations they have of their mother tongue and foreign languages. Findings show that teachers play an important role in the construction of representations and that these evolve throughout the learning process.

Key words: foreign language, mother tongue, plurilingual and pluricultural competence.

\section{Introducción}

Uno de los problemas que los estudiantes de pueblos ancestrales deben afrontar es el acceso a la educación superior. En consecuencia, la Universidad de Antioquia expidió en 2002 el acuerdo 236 por medio del cual se asignaron cupos específicos a la población proveniente de comunidades indígenas y afrocolombianas. El acuerdo busca, a través de medidas de discriminación positiva, favorecer la inclusión y el acceso a la universidad a dichas poblaciones, quienes han sido históricamente marginalizadas. Es así como, desde el año 2003, el programa de Licenciatura en Lenguas Extranjeras ha recibido 32 estudiantes que han ingresado bajo la modalidad "estudiante indígena". Sin embargo, las estadísticas del Departamento de Admisiones y Registro revelan un $40 \%$ de deserción de los estudiantes provenientes de tales comunidades. Algunos han desertado por bajo rendimiento académico, otros por razones que desconocemos. Por este motivo un grupo de investigadores del grupo de investigación en Enseñanza y Aprendizaje de Lenguas Extranjeras (EALE), de la Escuela de Idiomas de la Universidad de Antioquia, decidió explorar los retos académicos, lingüísticos y sociales en el aprendizaje de lenguas extranjeras que enfrentan los estudiantes indígenas activos en dicho programa. Varias categorías surgieron del análisis de los datos: experiencias educativas a nivel escolar y universitario; retos académicos y sociales; implicaciones para la Escuela de Idiomas; identidad cultural, étnica y lingüística; y representaciones de las lenguas. Esta última categoría corresponde a uno de los objetivos específicos del proyecto: explorar las representaciones que los estudiantes indígenas de la licenciatura tienen sobre las lenguas que hacen parte de sus repertorios lingüísticos y su influencia en el aprendizaje de las lenguas extranjeras que estudian, inglés y francés.

\section{Situación y políticas lingüísticas en Colombia}

Según la Constitución Política de 1991 (artículos 7 y 10), Colombia es un país cultural y lingüísticamente diverso. De esta manera, la carta magna reconoce y protege la legitimidad de los pueblos indígenas y criollos y de sus lenguas. Adicionalmente, se señala que los pueblos indígenas recibirán una educación bilingüe intercultural en sus territorios. Por otra parte, La Ley 1381 de 2010, conocida como la ley de Lenguas Nativas y publicada por el Congreso de la República, expide las normas sobre "reconocimiento, fomento, protección, uso, preservación y fortalecimiento de las lenguas de los grupos étnicos de Colombia y sobre sus derechos lingüísticos y los de sus hablantes" (p.1). Una lengua indígena es, según Pachón y Correa (1997), aquella que se considera típica de los habitantes oriundos de una determinada región. Según tales autores, en el caso de América Latina se ha acuñado el término para referirse a las lenguas empleadas por las comunidades que habitaban el continente antes de la llegada de los conquistadores europeos. González de Pérez (2011, p. 31) señala que una lengua indígena es un "sistema de comunicación estructurado de forma coherente", por lo tanto, estas lenguas no son dialectos y el uso de este término es incorrecto.

Actualmente, existen en Colombia alrededor de 68 lenguas habladas además del español, lengua materna de la mayoría de la población (González de Pérez, 2011; Landaburu, 2012; Patiño Rosseilli, 2000, 1992). Dentro de estas cifras se cuentan el criollo hablado en San Andrés y Providencia (Bartens, 2003; Dittmann, 2012); el criollo hablado en San Basilio de Palenque (Patiño Rosselli, 2012); la lengua romaní de los pueblos gitanos (González de Pérez, 2011); y 65 lenguas amerindias habladas por los diferentes pueblos indígenas del país. Ardila 
(2012) señala que según el último Censo Nacional la población indígena es de 1.392.623, la cual corresponde al 3,4\% de la población total del país. Por su parte, Landaburu (2012) explica que según este censo, se reconocen cerca de 100 identidades étnicas que no corresponden en su totalidad a identidades etnolingüísticas, pues alrededor de 550.000 personas no hablan ninguna lengua indígena. La totalidad de hablantes de las lenguas indígenas es, según este autor, de 800.000 , lo cual equivale a menos del $2 \%$ de la población colombiana.

\section{Estado del arte}

En Colombia se han realizado muy pocos estudios relacionados directamente con el bilingüismo o plurilingüismo de estudiantes de educación superior provenientes de comunidades indígenas. La revisión de la literatura nos arrojó algunos estudios, uno en contexto de educación secundaria y otros en contextos universitarios. En el primero, Jaraba y Arrieta (2012) exploran el aprendizaje del inglés por parte de un grupo de estudiantes de la comunidad Zenú. Gracias a una investigación-acción en la cual las maestras integraron en la clase de inglés actividades propias de esta cultura, los estudiantes encontraron razones significativas para el estudio de la lengua inglesa. En cuanto al contexto universitario, encontramos, por una parte, el estudio de Cuasilpud (2010), quien se enfocó en las actitudes de dos estudiantes indígenas hacia al aprendizaje del inglés al tomar un curso en la modalidad virtual. Los resultados de este estudio sugieren la necesidad de crear tutorías para los estudiantes indígenas que toman cursos de lengua, no solo en esta modalidad sino también en la presencial, con el fin de acompañarlos en su proceso de adaptación al contexto universitario y de motivarlos al aprendizaje de la lengua. Por otra parte,Velandia (2007) reporta un estudio de caso en el que se ofreció un plan de acompañamiento a un estudiante de la comunidad Inga, con el fin de favorecer el mejoramiento de su habilidad oral en inglés. Las conclusiones de este estudio sugieren continuar la investigación en esta área en Colombia, entre otras razones, porque en nuestro país "se ha prestado poca atención al multilingüismo debido a la expansión de la globalización; algunos miembros de las comunidades indígenas están utilizando tres o más idiomas; el español, su lengua indígena y una lengua extranjera" (p.130). Merece la pena señalar un estudio llevado a cabo en las universidades Javeriana, Distrital y Nacional de Colombia en la ciudad de Bogotá, acerca de la escritura académica de un grupo de estudiantes indígenas y afrocolombianos de aquellas universidades matriculados en diferentes programas académicos (Soler, 2013a; Agray, 2014). $\mathrm{Al}$ respecto, Soler (2013b) concluye que la situación de dichos estudiantes en la universidad es compleja y por lo tanto es necesario buscar estrategias para transformar la universidad en un espacio plural que vayan "más allá de garantizar el acceso a la universidad de estas poblaciones" (p.78). La revisión de estas investigaciones nos muestra que no existen hasta el momento estudios documentados que aborden el aprendizaje de dos lenguas extranjeras (aparte del español), de manera simultánea por parte de los estudiantes pertenecientes a comunidades de pueblos nativos.

\section{Fundamentos conceptuales}

Estudiar la relación que un sujeto establece con las diferentes lenguas que habla y estudia implica explorar la noción de representaciones sociales, que abordaremos en un primer lugar. Posteriormente, a fin de caracterizar la situación lingüística de un sujeto que habla varias lenguas, analizaremos la noción de competencia plurilingüe y pluricultural.

\section{Representaciones sociales}

La noción de representaciones sociales, propuesta inicialmente en los años 60 por el psicólogo social Serge Moscovici, ha sido abordada desde diversos ámbitos del conocimiento (Araya, 2002; Stratilaki, 2011). Según Stratilaki (2011) su investigación se ha hecho cada vez más frecuente en la didáctica de las lenguas. Su carácter multidisciplinario la convierte en una noción compleja, polisémica y variable, la cual no se inscribe dentro de una disciplina específica, en tanto toca todos los aspectos de la vida social. Así, existe una gran diversidad de teorías, métodos de estudio y análisis de las representaciones sociales (Stratilaki, 2011). 
Para fines del presente artículo, adoptaremos la definición propuesta por Araya (2002) quien entiende las representaciones como

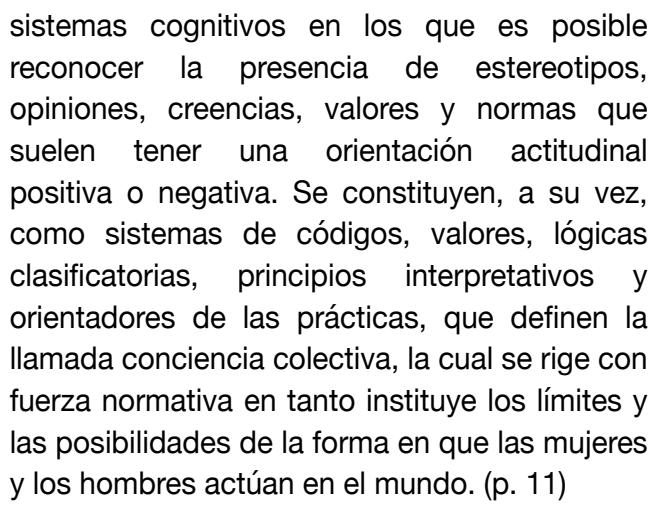

Por su parte, Rubenfeld, Clément, Lussier, Lebrun, $\mathcal{E}$ Auger (2006) sugieren que las representaciones sociales se forman a través del discurso y la interacción. Asimismo, Araya (2002, p. 9) afirma que se trata de una noción

que unifica e integra lo individual y lo colectivo, lo simbólico y lo social; el pensamiento y la acción", lo cual complejiza la noción de representación. De este modo, el individuo elabora sus representaciones "en función de sus imágenes interiores, pero también de la historia de su vida, de su identidad lingüística y de su lugar en el contexto social. (2011, p. 39)

Por su parte, Guimelli (1994, en Stratilaki, 2011, p. 34) explica que una representación social tiene un carácter dinámico debido a su constante evolución en función de contextos sociales e históricos distintos. Así, son negociadas y renegociadas en las interacciones sociales.

En el ámbito lingüístico, Castellotti y Moore (2002) sostienen que las representaciones influyen en el aprendizaje y utilización de las lenguas. Las mismas autoras añaden que numerosos estudios muestran la gran influencia de las representaciones de los aprendices sobre el proceso de aprendizaje de las lenguas, ya que pueden reforzarlo y valorizarlo o, por el contrario, inhibirlo. Dichas representaciones pueden concernir las normas y características de las lenguas, sus hablantes y su estatus con respecto a otras lenguas.

En las prácticas lingüísticas, las representaciones van más allá de las lenguas en sí, en la medida en que implican las relaciones con los otros, el posicionamiento en el mundo y la identidad. En tanto objeto de discurso, estas adquieren una dimensión observable que permite su estudio y sistematización (Moore, 2001).

Las representaciones tienen la capacidad de dirigir nuestros comportamientos, así como la forma en la que establecemos relaciones con otros (Rubenfeld, Clément, Lussier, Lebrun, \& Auger, 2006). Sin embargo, a pesar de la relación que se ha establecido entre representaciones y prácticas, Billiez y Millet (2001) afirman que la influencia de las primeras en el actuar y en las prácticas lingüísticas es compleja y a veces contradictoria.

\section{Sujeto plurilingüe, competencia plurilingüe y pluricultural}

Kramsch (2009) define el sujeto plurilingüe como aquel que utiliza, en su cotidianidad, más de una lengua. En esta definición se incluyen también los aprendices de lenguas extranjeras e incluso los usuarios que ella llama "silenced speakers", quienes comprenden una lengua, pero no la hablan por diversas razones; por ejemplo las comunidades a las que se les prohibió el uso de su lengua en la escuela pero seguían escuchándola en su comunidad, desarrollando solo la capacidad de comprensión. Por su parte, Grosjean (2010) arguye en sus distintos trabajos que "es bilingüe quien usa dos o más lenguas (o dialectos) en su vida diaria" (2010, p.4); adopta de este modo una perspectiva funcional del bilingüismo. Desde este enfoque, el término bilingüismo caracteriza también a quienes hablan más de dos lenguas, como es el caso de los participantes de este estudio.

Ahora bien, el sujeto plurilingüe es poseedor de una competencia plurilingüe y pluricultural definida por el Instituto Cervantes (2002) como

la capacidad de utilizar las lenguas para fines comunicativos y de participar en una relación 
intercultural en que una persona, en cuanto agente social, domina-con distinto grado-varias lenguas y posee experiencia de varias culturas. Esto no se contempla como la superposición o yuxtaposición de competencias diferenciadas, sino como la existencia de una competencia compleja e incluso compuesta que el usuario puede utilizar. (p.167)

Según Castellotti y Moore (2011), este concepto se impone, a mediados de los años noventa, como un referente esencial para pensar la relación a la pluralidad y a la diversidad en las situaciones de apropiación de las lenguas. Este, sin embargo, se desarrolla notablemente en el transcurso de la década siguiente con la publicación del Marco Común Europeo de Referencia para las Lenguas. Inicialmente los trabajos científicos se inspiraron en la sociolingüística de contacto, después fueron enriqueciéndose con otros marcos de análisis, tales como el estudio de historias de vida y las biografías lingüísticas. Del mismo modo, comenzó a desarrollarse un interés particular por grupos hasta ese entonces marginalizados en los estudios, como los migrantes y los hablantes de lenguas minoritarias o minorizadas.

Tanto para Coste, Moore, y Zarate (1997, p.11) como para los autores del Marco de Referencia, esta noción concibe lo lingüístico y lo cultural como dos componentes estrechamente relacionados, inscritos dentro de una lógica socioidentitaria fuertemente marcada por la alteridad, donde el individuo es visto como un actor social con capacidad de adaptarse a las circunstancias y responder a necesidades comunicativas. La competencia plurilingüe y pluricultural es concebida como el resultado de trayectorias familiares, comunitarias y sociales. Esta se caracteriza por ser singular y única, pero al mismo tiempo dinámica y evolutiva en el tiempo y en el espacio. En este sentido la competencia plurilingüe y pluricultural no es la simple adición de competencias monolingües o la yuxtaposición de competencias distintas sino el resultado de intercambios, de mediaciones entre actores de varias lenguas y culturas bajo formas y combinaciones plurales que evolucionan (Beacco et al., 2010; Coste, Moore, \& Zarate, 1997; InstitutoCervantes, 2002).

\section{Metodología}

Este estudio se enmarcó dentro de los principios de la investigación cualitativa, particularmente dentro del enfoque de estudio de caso (Creswell, 2007; Gagnon, 2012). Según Gagnon (2012), un estudio de caso "permite una comprensión profunda de los fenómenos, de los procesos que los componen y de las personas que hacen parte de él" (nuestra traducción, p.2). Optamos por un enfoque cualitativo de tipo exploratorio y descriptivo, motivados por el interés de comprender a profundidad la forma en que los participantes del estudio vivían su realidad como aprendices de lenguas en la Universidad de Antioquia.

\section{Participantes}

Participaron de este estudio los estudiantes admitidos al programa de Licenciatura en Lenguas Extranjeras bajo la modalidad de estudiante indígena. Al momento de comenzar la investigación, el programa contaba, según las estadísticas del Departamento de Admisiones y Registro, con dieciséis estudiantes activos. Dichos estudiantes fueron contactados por el equipo de investigación, de los cuales ocho aceptaron participar. Siguiendo el protocolo ético, los participantes firmaron un formato de consentimiento. El grupo estuvo constituido por ocho estudiantes matriculados entre el primero y el octavo semestre; cinco mujeres y tres hombres provenientes de las comunidades: Misak (Guambiano), Gunadule (Tule), EmberáChamí, y Zenú. Tres de los estudiantes tenían una lengua materna indígena; para los cinco restantes el castellano era su lengua materna. Igualmente, participó en la investigación un grupo de profesores de inglés y francés que tenía a su cargo los cursos en los cuales se encontraban inscritos los estudiantes participantes del estudio. En la tabla 1 presentamos la información de los participantes. ${ }^{5}$

\footnotetext{
5 Con el fin de proteger la identidad de los estudiantes participantes, identificamos a cada uno de ellos con un número. No proveemos información sobre su sexo, sus comunidades ni el nombre de sus lenguas maternas pues al ser tan pocos, podrían ser identificados fácilmente por un lector que los conozca.
} 
Tabla 1. participantes del estudio y sus situaciones lingüísticas

\begin{tabular}{|c|c|c|}
\hline \multirow[t]{2}{*}{ Estudiante } & \multicolumn{2}{|c|}{ Situación lingüística } \\
\hline & Lengua(s) materna(s) & Lenguas extranjeras \\
\hline 1 & $\begin{array}{c}\text { L16: Español } \\
\text { L2: Lengua indígena }\end{array}$ & $\begin{array}{l}\text { L3: Francés } \\
\text { L4: Inglés }\end{array}$ \\
\hline 2 & $\begin{array}{l}\text { L1: Lengua indígena } \\
\text { L2: Español }\end{array}$ & $\begin{array}{l}\text { L3: Inglés } \\
\text { L4: Francés }\end{array}$ \\
\hline 3 & $\begin{array}{l}\text { L1: Lengua indígena } \\
\text { L2 : Español }\end{array}$ & $\begin{array}{l}\text { L3: Inglés } \\
\text { L4: Francés }\end{array}$ \\
\hline 4 & Español & $\begin{array}{l}\text { L2: Inglés } \\
\text { L3: Francés } \\
\text { L4: Portugués } \\
\text { L5: Alemán }\end{array}$ \\
\hline 5 & Español & $\begin{array}{c}\text { L2: Inglés } \\
\text { L3: Francés } \\
\text { L4:Conocimientos básicos de lengua indígena }\end{array}$ \\
\hline 6 & Español $^{7}$ & $\begin{array}{l}\text { L2: Inglés } \\
\text { L3: Francés }\end{array}$ \\
\hline 7 & Español & $\begin{array}{l}\text { L2: Inglés } \\
\text { L3: Francés }\end{array}$ \\
\hline 8 & Español & $\begin{array}{l}\text { L2: Inglés } \\
\text { L3: Francés }\end{array}$ \\
\hline
\end{tabular}

Fuente: elaboración propia.

\section{Técnicas de recolección de datos}

La recolección de la información se realizó por medio de las siguientes fuentes:

Entrevistas a los estudiantes: la primera fuente de datos utilizada fue una entrevista individual de tipo comprensivo (Billiez y Millet, 2001, p.41) y biográfico a cada uno de los participantes. Nos interesaba explorar las biografías lingüísticas (Delas, 2006; Molinié, 2006) de los estudiantes por medio de sus relatos de vida. El análisis de estas entrevistas nos permitió identificar en cinco de los participantes una filiación identitaria muy fuerte con sus comunidades. Para las siguientes fuentes de datos, decidimos profundizar en estos cinco casos, de los cuales tres tenían una lengua indígena como lengua materna.

6 Este dice: La letra "L" se refiere a lengua y el número al orden de adquisición

7 En los participantes 6,7 y 8 no se evidenció una filiación identitaria fuerte con sus comunidades a pesar de haber sido admitidos en la universidad como indígenas. El no asumirse como indígenas sumado al hecho de que no poseían una lengua materna indígena nos condujeron a no profundizar el análisis de estos tres casos.
Un día en la vida de: implementamos observaciones de clase bajo esta modalidad en la cual un investigador acompañaba a cada uno de los estudiantes participantes durante un día de clases en la universidad, con el objetivo de comprender cómo transcurría su cotidianidad en una jornada académica.

Grupos focales: después de realizar la primera entrevista y las observaciones, condujimos dos grupos focales (Dendinger, 2000) con los profesores que tenían a cargo los cursos en los que estaban matriculados los estudiantes, con miras a obtener sus perspectivas sobre la presencia de estudiantes indígenas en sus clases. En total, participaron ocho profesores, cinco de inglés y tres de francés. Dichos profesores tenían a su cargo cursos de naturaleza diferente, algunos básicos de inglés o francés y otros del ciclo avanzado, de contenido específico dictados en inglés o francés como Literatura, Diseño de Cursos y Materiales, Proyecto Pedagógico, entre otros.

Entrevistas a profundidad: al cabo de seis meses de haber realizado la primera entrevista individual, efectuamos dos entrevistas colectivas a profundidad 
con los cinco estudiantes del grupo seleccionado después de la primera entrevista.

Documentos oficiales: recurrimos a algunos datos provenientes de fuentes oficiales como las estadísticas provistas por Admisiones y Registro y la legislación de la universidad con respecto al tema.

\section{Procedimientos para análisis de datos}

El análisis de los datos se realizó de forma cualitativa por medio de la creación de categorías emergentes (Creswell, 2007; Huberman \& Miles, 1991). Todas las entrevistas y grupos focales fueron transcritos. En una segunda etapa, realizamos un análisis del discurso (Benveniste, 1974; KerbratOrecchioni, 1980) de las entrevistas. Este segundo análisis no aparece documentado en este artículo. Con miras a validar la información, recurrimos a la triangulación desde las siguientes perspectivas propuestas por Burns (1999): en el tiempo (recogiendo los datos en diferentes momentos de la investigación); en el espacio (datos recogidos de diferentes grupos, en este caso de estudiantes y profesores); y, desde los investigadores (docentes, estudiantes, observadores). La organización y análisis de la información se realizó con la ayuda del programa NVIVO 10.

\section{Resultados}

El análisis de los datos nos permitió develar las biografías lingüísticas de los estudiantes participantes del proyecto y sus representaciones sobre las lenguas. Por lo tanto, presentaremos los repertorios lingüísticos de los estudiantes y abordaremos las representaciones de las lenguas maternas y extranjeras.

\section{Los repertorios lingüísticos de los estudiantes}

Pudimos establecer dos perfiles lingüísticos. Así, de los ocho estudiantes participantes, solo tres hablaban una lengua indígena (Tule, Emberá-chamí y Guambiano) provenientes de los departamentos de Antioquia y Cauca. Dos de ellos adquirieron simultáneamente su lengua indígena y el español durante su infancia. Uno de los estudiantes adquirió primero el castellano y luego su lengua indígena. Para estos estudiantes, el inglés es la primera lengua extranjera y el francés la segunda; completando un repertorio de cuatro lenguas con las que interactúan en su vida diaria. Los cinco estudiantes restantes tenían el español como lengua materna, ya que el idioma original desapareció de sus comunidades. Todos ellos comenzaron el aprendizaje del inglés como lengua extranjera en el colegio y posteriormente del francés en la universidad. Uno de ellos tenía conocimientos básicos de lengua emberá pues había intentado estudiarla y otro, además de las lenguas mencionadas, estudiaba alemán y portugués en uno de los programas de la universidad.

\section{Representaciones de las lenguas}

\section{Lenguas maternas}

Entre los estudiantes se distinguen entonces dos grupos: aquellos que hablan una lengua indígena, la cual es en la mayoría de los casos lengua materna $\mathrm{y}$ aquellos que tienen el español como lengua materna, pero en cuya comunidad se reconoce haber tenido una lengua vernácula que se perdió.

\section{Lenguas indígenas}

Los hablantes de lenguas indígenas tienen una actitud muy positiva hacia sus lenguas maternas. Esta valoración va acompañada de comentarios como:

Para mí [mi lengua] significa riqueza totalmente.

(Estudiante 3, entrevista individual)

Algo maravilloso. (Estudiante 2, entrevista individual).

De esta forma, los participantes hablantes de lenguas indígenas le atribuyen a sus lenguas nativas una connotación afectiva como algo íntimo y perteneciente a la familia. También las reconocen dentro de una tradición étnica, como lo explica el estudiante 3:

Para mí [mi lengua] pues significa todo. En parte porque tengo la conexión con mis papás y mis abuelos. Eh, me da como la identidad, desde 
donde soy por lo que pues, soy [indígena]. (entrevista individual)

Algo que percibimos al preguntar a los estudiantes indígenas por el uso de sus lenguas es la conciencia que tienen de que se están extinguiendo y se sienten responsables, como hablantes de sus lenguas, de utilizarlas y transmitirlas a las nuevas generaciones. Esto se evidencia en el siguiente testimonio:

Ojalá la conserváramos [...] porque si uno deja de hablar una lengua, es una forma de extinción, lo que sucede con los Zenúes, que ellos ya no tienen su propia lengua. (Estudiante1, entrevista individual)

Al respecto, la construcción de la identidad como indígena reposa para estos estudiantes sobre una identificación territorial y cultural específica, en la cual la lengua es el elemento central. Los siguientes testimonios sustentan esta idea:

para mí ser indígena, como dicen las compañeras, es la comunidad, el territorio [...] eh, las costumbres, pero principalmente más es la lengua porque con ello, por lo menos viví un tiempo con mi abuela y con ella aprendí muchas cosas y a través de sus historias, de sus [...] de su vida pues uno aprende [...] aprende [...] uno [...] tanto de lo que ha olvidado cuando uno sale al colegio y todo eso (Estudiante 3, entrevista grupal) [énfasis agregado].

La actitud valorativa que los estudiantes tienen de sus lenguas y comunidades se refleja en su posicionamiento en las clases de inglés y francés, como lo deja entrever el siguiente comentario "yo digo que se sentía pues como muy orgullosa y lo hacía muy evidente en ella de, de pertenecer a una comunidad indígena" (Profesor 1, grupo focal 1)

En el caso de los estudiantes que no hablan una lengua indígena, identificamos varios fenómenos que explican la ausencia de la lengua ancestral. Para algunos, se trata de comunidades que, por razones históricas, que se remontan a la época de la colonia, perdieron su lengua. En otros casos, la lengua de la comunidad sigue viva, pero no en los resguardos de los que provienen los estudiantes. Un tercer caso se refiere a estudiantes cuyos padres se alejaron de sus comunidades de origen y no perpetuaron la lengua en la generación naciente. Cabe resaltar que estos estudiantes le atribuyen a las lenguas de sus ancestros un fuerte valor simbólico, se reconocen y se reivindican como indígenas por sus costumbres y pertenencia a sus comunidades. Algunos incluso expresan pesadumbre por no hablar una lengua indígena y porque se perdió en su comunidad, como lo afirma el estudiante 4 al describir su relación con la lengua materna

yo veo el español como, no es mi lengua nativa, o sea no la considero como mi lengua nativa porque se supone que mi lengua nativa es el [lengua indígena]. (Estudiante 4, entrevista individual) [énfasis agregado].

La ausencia de la lengua indígena se convierte en ocasiones en una causa de discriminación por parte de otras comunidades que han conservado sus lenguas e incluso del gobierno. Es importante subrayar, sin embargo, que en algunas comunidades la lengua nativa sigue siendo usada únicamente por los médicos tradicionales durante los rituales que se efectúan.

Por otra parte, algunos manifiestan pesadumbre al sentir que por el contexto en el que se desenvuelven actualmente y en el cual el español es la lengua de comunicación, se les hace más difícil practicar su lengua materna o sienten que tienen dificultades para expresar ciertas ideas, como lo plantea uno de los participantes:

me da mucho miedo que yo pierda lo que soy. $A$ veces siento ya [mi idioma] ya no lo domino muy bien como antes. (Estudiante 1, entrevista individual)

Finalmente, para este grupo de estudiantes, sus lenguas indígenas son el punto de referencia al aprender otras lenguas.

\section{La lengua española}

El análisis de las entrevistas nos permitió develar que algunos estudiantes adscriben una fuerte 
filiación identitaria a sus comunidades mientras que otros no se posicionan como indígenas en sus discursos. En efecto, el primer grupo de estudiantes ve el español como algo externo a sus comunidades que les permite establecer contacto con las demás personas; es decir que lo emplean con fines comunicativos e instrumentales contrariamente a la afectividad asociada a las lenguas indígenas. Al respecto, los siguientes estudiantes manifestaron que el español es "otra forma de poder expresarme con, pues con personas que ya no son del resguardo" (Estudiante 3), "lo de afuera, si, es eso, el entorno de afuera" (Estudiante 2).

Por su parte, el segundo grupo de estudiantes le atribuye al español un valor más afectivo en el sentido de que se trata de su lengua de comunicación con la familia y la comunidad, así como con el exterior. Además, para este grupo de estudiantes el español es la lengua de referencia para aprender otras.

\section{Lenguas extranjeras}

\section{La lengua inglesa}

Las respuestas obtenidas indican que el inglés es visto como la lengua que permite una apertura al mundo, a los viajes, a estudios en el extranjero y al acceso a la información. Es además percibida como una lengua relacionada con el conocimiento ya que tiene mayor difusión y por ende tienen más oportunidades para practicarla en su cotidianidad.

Las representaciones sobre la lengua inglesa varían entre los estudiantes que apenas están iniciando su proceso de formación en la universidad y quienes ya lo están culminando. Los primeros hacen más alusión a su estructura, la cual es percibida por algunos como simple, mientras que los segundos amplían el concepto de lengua y lo relacionan con sus hablantes y culturas. En ambos casos, el inglés es asociado, al inicio de los estudios universitarios, con un sentimiento de frustración. Esto se explica porque en sus primeras clases de inglés, los estudiantes indígenas descubren que su nivel es muy bajo en comparación con el de sus compañeros. De acuerdo a los estudiantes, pareciera que los profesores de inglés que imparten cursos de nivel 1 asumen que todos los estudiantes ingresan a la universidad con conocimientos sólidos en dicha lengua. Por esta razón, se identifica el inglés como un idioma que en el inicio del aprendizaje ha dejado experiencias de fracaso y de poca motivación en los participantes del proyecto. Esta posición es compartida por todos los estudiantes y se manifiesta claramente en los siguientes testimonios:

\begin{abstract}
Me acuerdo mucho en el primer semestre que yo no entendía absolutamente nada de lo que el profesor decía y solo cuando decía [mi nombre] [...] yo me asustaba porque no entendía lo que me estaba diciendo y todos se reían, y eso [...] lo hace a uno estresar, cada vez que yo iba a esa clase.......] él como por hacerme participar entonces todas las clases siempre que yo llegaba ahí mismo la pregunta para mí, y eso me estresaba mucho. (Estudiante 5, entrevista individual)

Pues mi inglés era gramática de colegio que uno va y dice como ah, para qué me sirve esto, [...]. Y fue muy duro para mí el curso oral porque la Escuela asume que uno viene de colegios [...] como los demás, que vienen de colegios que le hablan- le dan las clases en inglés y uno no. [...] ah, sí, yo podía dar inglés pero en [mi lengua indígena], un poquito en [mi lengua indígena] y el otro pedazo en español porque ni en inglés te lo dan. (Estudiante 2, entrevista individual)
\end{abstract}

Las primeras experiencias con el inglés en la universidad forjan una preferencia inicial hacia la lengua francesa debido a que la mayoría de estudiantes del programa comienza de cero su aprendizaje; esta situación permite que los estudiantes indígenas se sientan en igualdad de condiciones con respecto a sus compañeros al aprender esta lengua. Sin embargo, esta preferencia puede cambiar a lo largo del proceso y en muchos casos el inglés termina siendo la lengua de predilección porque les brinda más oportunidades profesionales y de desarrollo de proyectos futuros en sus respectivas comunidades, por ejemplo, dando a conocer sus comunidades en diversos medios en la lengua inglesa.

Los discursos de los docentes permiten constatar que las representaciones sobre la 
lengua inglesa se ven reflejadas en el actuar de los estudiantes durante las clases:

Primero el francés es su idioma favorito. Eh, en inglés ella se sentía como...muy asustada, en ese grupo fue ganando confianza [...] porque el inglés no era su idioma favorito y ahí en ese curso logramos que por lo menos medio [...] lo acogiera con cariño. (Profesor 1, grupo focal 1)

\section{La lengua francesa}

Todos los estudiantes mostraron una disposición positiva hacia el francés. La mayoría destaca su belleza haciendo alusión a su sonoridad y atribuyéndole cierta connotación de lengua romántica. Además, la mayoría la considera como una lengua compleja y en muchos casos este aspecto constituye un reto más que un obstáculo.

Como lo mencionamos anteriormente, a diferencia del inglés, la mayoría de estudiantes del programa comienzan francés sin muchos conocimientos previos. Esto se convierte en una ventaja en el proceso de aprendizaje generando tranquilidad en los estudiantes indígenas. En efecto, pareciera que el francés les permite integrarse más fácilmente a sus compañeros debido a que pone en pie de igualdad a todos los estudiantes. Esto se evidencia en los siguientes testimonios:

Porque en francés aquí en la Escuela se empieza desde cero [...], en cambio en inglés asumen que todos ya tenemos una base sobre el inglés entonces empiezan [...] a hablar, a hablar y a hablar. (Estudiante 5, entrevista individual)

En el caso mío [...] me motivó saber que todos estábamos como igual, que vamos a empezar todos en un proceso de construcción, en cambio en el inglés como la compañera dice ya habían muchos compañeros que ya hablaban el idioma. (Estudiante 3, entrevista individual)

Esta misma diferencia es percibida por los profesores como una ventaja:

Pero yo creo que en francés si hay una diferencia y es, porque casi todos en francés eran debutantes [...] estaban todos en el mismo nivel. (Profesor 3, grupo focal 2)
Al mismo tiempo, se percibe una evolución en las representaciones sobre el francés. Por ejemplo, para algunos el francés era poco hablado en el mundo y por ende ofrecía pocas posibilidades académicas y profesionales; esta representación se transforma a medida que el individuo avanza en su formación. El francés pasa a ser para todos los estudiantes una lengua que también permite el acceso a la información y la apertura al mundo; así lo expresa el siguiente estudiante

\section{Eh, pues a mí me parece [el francés] algo como más pequeño. Pues, no sé, [...] pues que solamente lo hablan en Francia. Pero pues es lo, era lo que creía, pero no, ya no, vi que no es así. (Estudiante 8, entrevista individual)}

Finalmente, en dos casos, la lengua indígena es el punto de referencia para el aprendizaje del francés, ya que en sus lenguas maternas existen sonidos nasales que les permiten acercarse a la nasalización en lengua francesa.

\section{Discusión y Conclusiones}

A partir de los anteriores hallazgos, es necesario emitir algunos comentarios. En primer lugar, existen según Ardila (2012, p. 439) las siguientes tendencias en la competencia lingüística de los hablantes de lenguas indígenas en Colombia:

Mayores de 40 años: bilingües equilibrados o dominantes en lengua indígena. En pocos casos monolingües en lengua indígena.

- Hablantes entre 20 y 40 años: bilingües dominantes en español con competencia activa o pasiva en lengua indígena.

- Menores de 20 años: monolingües en español o bilingües con competencia pasiva en lengua indígena.

- Si comparamos los rangos de edad, los participantes del estudio se ubican en la segunda y mayoritariamente en la tercera categoría. 
En este sentido, nuestros hallazgos coinciden con otros estudios, como el reportado por Soler (2013a) quien explica que en los estudiantes indígenas universitarios en Bogotá D.C., "la competencia en lengua indígena es dispar" (p.50) pues no todos hablan una lengua indígena. Sin embargo, pareciera que de los hablantes de lengua indígena, uno se sitúa en la categoría 2 (bilingüe dominante en español) y dos en la categoría 1 (bilingües equilibrados) a pesar de no superar los 30 años. Con respecto a estos últimos, se observó el establecimiento de lo que Moore (2006) denomina "comunidades de práctica", es decir, grupos que posibilitan "mejorar las competencias individuales gracias al compromiso mutuo" (p.40). Este es el caso particular de los estudiantes que continúan utilizando sus lenguas para interactuar con otros estudiantes de sus comunidades o sus parientes durante su estadía en la ciudad con el fin de no perderlas. Estos estudiantes movilizan un capital lingüístico y cultural ligado a las diferentes lenguas que hacen parte de sus repertorios lingüísticos.

También es importante señalar que los estudiantes valoran positivamente sus lenguas maternas pues, a pesar del poco reconocimiento de estas en el medio, se consideran bilingües y ven a sus comunidades como bilingües. En este punto también es importante detenernos en la compleja noción de lengua materna. Para Skutnabb-Kangas (1988 citado por Moore, 2006, p. 108) existen cuatro criterios para definir una lengua materna: origen, competencia, función e identificación (interna o externa). En el caso del estudiante 1, podríamos aplicar el criterio de identificación interna, según el cual la lengua materna se refiere a la lengua con la que el individuo se identifica, pues a pesar de haber adquirido primero el español, él considera la lengua indígena como su lengua materna. Los participantes 2 y 3 siguen el criterio de origen pues consideran que su lengua materna es la que aprendieron en primer lugar, en este caso el idioma de sus ancestros. Merece la pena señalar el caso de un estudiante que no habla ninguna lengua indígena, pero quien identifica la lengua que se hablaba en su comunidad como su lengua materna a pesar de no poseer una competencia que le permita comunicarse en esta, siguiendo el principio de identificación interna.
Una lectura de los testimonios expuestos en la sección de representaciones sobre las lenguas indígenas, nos permite develar las tensiones identitarias que el factor lingüístico puede generar entre quienes hablan o no una lengua indígena. Es claro que para los hablantes de una lengua indígena, este hecho constituye su marca de identidad más fuerte, a pesar de hacer referencia también a sus identidades étnica y cultural. Incluso realizan comparaciones con quienes no hablan una lengua indígena, señalando este hecho como pérdida de una parte de su identidad. Es de notar en los mismos testimonios que aquellos que no tienen dominio de una lengua indígena tratan de atribuirle su identidad indígena a los factores étnicos y culturales, no sin generar tensión con quienes sí la hablan. Como lo diría Soler (2013a), los estudiantes indígenas "establecen una relación entre identidad y territorio" (p.50), pues este hace parte fundamental de su ser indígena cumpliendo una función cultural y política. Por otra parte, quienes tienen una lengua materna indígena experimentan una nueva tensión en el plano lingüístico debido al uso cotidiano del español en el medio urbano y de las lenguas extranjeras objeto de estudio; en otras palabras, la lengua que menos usan en su vida diaria es la que tiene un mayor valor afectivo para ellos. Adicional a esto, en las historias vida de estos estudiantes sobresale el deseo de conservar sus lenguas indígenas, pero al mismo tiempo la necesidad que ellos ven de alcanzar el mismo nivel de desempeño en español que el resto de la población monolingüe, para hacer frente a una necesidad social en un país cuya lengua dominante es el español. Uno de los estudiantes reportó, por ejemplo, haber sido discriminado en la escuela secundaria al compartir con compañeros no indígenas que se burlaban de su forma de hablar el español.

En segunda instancia, con respecto a las representaciones, los hallazgos confirman la influencia del profesor en la formación de las representaciones descrita en algunos estudios (Diab, 2009; Grijalva \& Esparza, 2013; Inozu, 2011). En efecto, el profesor de lenguas contribuye a la formación de las representaciones que sus estudiantes se hacen de las lenguas objeto de estudio por medio de los materiales y contenidos 
que propone en sus clases. En los hallazgos es claro que, al hacer referencia al inglés, en la gran mayoría de los casos, los estudiantes participantes lo relacionaban con Estados Unidos, Canadá, Inglaterra y Australia; igualmente el francés lo relacionaban solo con Francia. Esta situación refleja que las prácticas de los maestros en las clases de lengua privilegian materiales y temáticas provenientes de estos lugares. Llama la atención que, a pesar de tratarse de un grupo de estudiantes indígenas, sus representaciones sobre las lenguas extranjeras y las culturas hegemónicas asociadas a estas no difieren de las que expresan en los cursos de lenguas extranjeras los estudiantes universitarios de lenguas. Se esperaría que dichas representaciones fuesen diferentes debido a los diversos contextos de proveniencia. Sin embargo, ninguno de los participantes proviene de comunidades indígenas completamente aisladas del mundo occidental. Estamos entonces frente a una población bicultural cuyo contacto con la población no indígena está mediado por la lengua española, de la cual tienen un alto dominio. Este hecho les permitiría compartir con la población no indígena un universo de representaciones sobre las lenguas extranjeras influenciadas por la información que circula en los diferentes medios de comunicación y en los contextos escolares. Sería pertinente indagar si dichas representaciones variarían en una población indígena más alejada del mundo occidental.

Por otra parte, los datos nos confirman que, como lo han señalado los autores citados en los Fundamentos Conceptuales, las representaciones sobre las lenguas evolucionan. El ejemplo más claro en esta investigación es el caso del inglés. Al iniciar los estudios universitarios, los estudiantes indígenas llegaron motivados hacia esa lengua y con imágenes muy positivas de ella. No obstante, los primeros contactos con dicha lengua en la universidad fueron considerablemente negativos, pues el proceso de aprendizaje no fue tan fácil como ellos lo imaginaban, provocando un cambio de representaciones sobre dicho idioma. Más adelante, en el programa y una vez superada dicha dificultad, los estudiantes descubren la utilidad, especialmente en el campo laboral, de la lengua inglesa y el valor simbólico atribuido se modifica.
Con respecto a la influencia de las representaciones en el comportamiento, Billiez y Millet (2001, p.37) explican que las representaciones y el comportamiento están muy relacionados, pero se trata de una relación muy compleja puesto que en los intercambios se manifiestan todas las representaciones (de las lenguas en contacto, de la situación de comunicación, de sí mismo y del otro). Debido a esta complejidad, es difícil observar las representaciones en los comportamientos. Sin embargo, en esta investigación pudimos confirmar que, durante las clases de lenguas observadas, las costumbres y tradiciones culturales de los estudiantes se materializan en sus discursos y actividades de clase. Así la influencia de las representaciones positivas sobre sus comunidades y lenguas se refleja en su comportamiento en clase. También es claro que las representaciones sobre las lenguas extranjeras influencian la forma en que los estudiantes las aprenden. Así la experiencia de frustración vivida inicialmente con el inglés influencia positivamente el acercamiento a la lengua francesa.

En cuanto a las lenguas extranjeras objeto de estudio, es necesario aclarar que la relación que los estudiantes indígenas establecen con dichas lenguas, particularmente las tensiones generadas por la lengua inglesa, podría en algunos casos asemejarse a la que establecen otros grupos de estudiantes (otras minorías étnicas, estudiantes provenientes de zonas rurales o incluso de colegios urbanos con bajo nivel de formación en inglés). Aunque pueda coincidir con otras poblaciones, en este texto nos limitamos a exponer el caso de los estudiantes indígenas pues este fue el objeto de esta investigación. Sin embargo, se requieren otro tipo de estudios de corte más lingüístico para aclarar la influencia de las lenguas indígenas en el aprendizaje las lenguas extranjeras.

Con respecto al español, un aspecto poco explorado en esta investigación es el rol que dicha lengua tiene durante sus estudios universitarios. Los cursos pedagógicos son ofrecidos en español y pareciera que se convierten en un punto generador de confianza pues les permite, al inicio de sus estudios, expresarse más fácilmente que en inglés o francés. Además, por el tipo de contenido trabajado, 
logran establecer un vínculo con sus comunidades pues organizan sus trabajos pedagógicos alrededor de ellas.

Finalmente, esperamos que los hallazgos de esta investigación puedan inspirar otras reflexiones y estudios sobre este tema, poco explorado en nuestro país. Particularmente, investigaciones sobre la influencia de las lenguas indígenas en el aprendizaje de las lenguas extranjeras, así como sobre las diferentes textualidades de los pueblos ancestrales serían un valioso aporte a la discusión en este campo. Es igualmente significativo estudiar el papel que el español, como lengua segunda, juega en el aprendizaje de las lenguas extranjeras, así como en su adaptación a las prácticas académicas propias del contexto universitario y la relación de estas con sus comunidades de origen. También es importante proponer y evaluar programas de acompañamiento a la población indígena para brindarles herramientas de permanencia en el contexto universitario.

\section{Referencias}

Agray, N. (2014). (Coord.). Reflexiones sobre lengua, etnia y educación. Bogotá D.C.: editorialPontificia Universidad Javeriana.

Araya, S. (2002). Las representaciones sociales: ejes teóricos para su discusión. Costa Rica: FLACSO.

Ardila, O. (2012). Lenguas en Contacto en Colombia: una Perspectiva Histórica y Social.

En C. Patiño Rosselli y J. Bernal Leongómez (Eds.). El Lenguaje en Colombia (Vol. 1, pp. 431-445). Bogotá D.C.: Instituto Caro y Cuervo.

Bartens, A. (2003). A contrastive Grammar IslanderCaribbean Standard English-Spanish. Helsinki: Academia Scientiarum Fennica.

Beacco, J.-C., Byram, M., Cavalli, M., Coste, D., Cuenat, M. E., Goullier, F., \& Panthier, J. (2010). Guide pour le développement et la mise en ouvre de curriculums pour une éducation plurilingue et interculturelle. Strasbourg: Conseil de l'Europe.

Benveniste, É. (1974). L'appareil formel de l'énonciation. In É. Benveniste (Ed.). Problèmes de Linguistique Générale. Paris: Éditions Gallimard.

Billiez, J., E Millet, A. (2001). Représentations sociales: trajets théoriques et méthodologiques. In D. Moore (Ed.), Les représentations des langues et de leur apprentissage. Références, modèles, données et méthodes. France: Didier.

Burns, A. (1999). Collaborative Action Research for English Language Teachers: Cambridge University Press.

Castellotti, V., \& Moore, D. (2011). La compétence plurilingue et pluriculturelle : genèse et évolutions d'une notion-concept. In P. Blanchet \& P. Chardenet (Eds.), Guide pour la recherche en didactique des langues et des cultures (pp. 241-249). France: Éditions des archives contemporaines.

Castellotti, V., \& Moore, D. (2002). Représentations sociales des langues et enseignements. Retrieved from http://www.coe.int/t/dg4/linguistic/source/ castellottimoorefr.pdf

Colombia. (1991). Constitución Política. Bogotá D.C.: Legis.

Colombia. (2010). Ley 1381. Bogotá D.C., Colombia. Recuperado de: http://goo.gl/wFFmrA

Coste, D., Moore, D., \& Zarate, G. (1997). Compétence Plurilingue et Pluriculturelle (version revisée). Strasbourg: Éditions du Conseil de l'Europe.

Creswell, J. (2007). Qualitative inquiry and research design. Choosing among five Approaches (2 ed.). USA: Sage Publications, Inc.

Cuasilpud, R. (2010). Indigenous Students' Attitudes towards Learning English through a Virtual Program: A Study in a Colombian Public University. Profile Issues in Teachers' Professional, 12(2), 133-152.

Delas, D. (2006). Instances du sujet et travail en biographie langagière. Le français dans le monde. Recherches et applications, (39), 12-17.

Dendinger, M. (2000)How to organize a focus group. Meetings and conventions. Recuperado de http:// www.meetings-conventions.com/Resources/PlannerBasics/Back-to-Basics/HOW-TO-ORGANIZE-AFOCUS-GROUP/.

Diab, R. L. (2009). Lebanese EFL Teachers' Beliefs about Language Learning. TESL Reporter, 42(2), 13-34.

Dittmann, M. (2012). Lengua y Sociedad Criolla Anglófona en el Archipiélago de San Andrés. In C. Patiño Rosselli \& J. Bernal Leongómez (Eds.). El Lenguaje en Colombia (Vol. 1, pp. 715-761). Bogotá D.C.: Instituto Caro y Cuervo

Gagnon, Y. (2012). L'Étude de cas comme méthode de recherche (2 ed.). Quebec, Canada: Presses de l'Université du Québec.

González de Pérez, M. (2011). Manual de divulgación de las lenguas indígenas de Colombia. Bogotá D.C.: Publicaciones del instituto Caro y Cuervo 
Grijalva, S. D. C., \& Esparza, E. R. (2013). Pre-Service Teachers' Beliefs about Language Teaching and Learning: A Longitudinal Study. Profile, 15(1), 81-95.

Grosjean, F. (2010). Bilingual, Life and Reality. Cambridge: Harvard University Press.

Huberman, M., E Miles, M. (1991). Analyse des données qualitatives. Bruxelles: De Boeck.

Inozu, J. (2011). Beliefs about foreign language learning among students training to teach English as a foreign language. Social Behavior $E$ Personality: An International Journal, $\quad$ 39(5), 645-653.

Instituto-Cervantes. (2002). Marco común europeo de referencia para las lenguas: aprendizaje, enseñanza, evaluación. Madrid: Ministerio de Educación, Cultura y Deporte.

Jaraba, D., \& Arrieta, A. (2012). ETNOENGLISH: Trenzando palabras, historias y proyectos de vida del pueblo Zenú. Íkala, revista de lenguaje y cultura, 17(1), 89- 98.

Kerbrat-Orecchioni, C. (1980). Lénonciation. De la subjectivité dans le langage. Paris: Armand Colin.

Kramsch, C. (2009). The Multilingual Subject: Oxford University Press.

Landaburu, J. (2012). Tesoro indígena colombiano en riesgo. El Tiempo. Recuperado de http://www. eltiempo.com/archivo/documento/CMS-11983681

Molinié, M. (2006). Activité biographique et développement du sujet plurilingue : des acquis méthodologiques aux questions de formation. Le français dans le monde. Recherches et applications, 39, 171-189.

Moore, D. (2001). Les représentations des langues et de leur apprentissage: itinéraires théoriques et trajets méthodologiques. In Moore, D. (Ed.) Les représentations des langues et de leur apprentissage: références, modèles, données et méthodes. France: Didier.
Moore, D. (2006). Plurilinguismes et école. Paris: Editions Didier.

Pachón, X., E Correa, F. (1997). Lenguas amerindias, condiciones socio-lingüísticas en Colombia. Santa Fe de Bogotá, Colombia: Imprenta Patriótica del Instituto Caro y Cuervo.

Patiño Rosselli, C. (2012). El Idioma Criollo de San Basilio de Palenque. In C. Patiño Rosselli \& J. Bernal Leongómez (Eds.). El Lenguaje en Colombia (Vol. 1, pp. 697-715). Bogotá D.C.: Instituto Caro y Cuervo

Patiño Rosseilli, C. (2000). Sobre etnolingüística y otros temas. Bogotá D.C.: Instituto Caro y Cuervo.

Patiño Rosseilli, C. (1992). La criollística y las lenguas de Colombia. Thesaurus, XLVII (2), 233-264.

Rubenfeld, S., Clément, R., Lussier, D., Lebrun, M., \& Auger, R. (2006). Second Language Learning and Cultural Representations: Beyond Competence and Identity. Language Learning, 56(4), 609-631. doi: 10.1111/j.1467-9922.2006.00390.x

Soler, S. (2013a). Usted ya en la universidad y no sabe escribir. Escritura y poder en la universidad. Bogotá D.C.: Universidad Distrital Francisco José de Caldas.

Soler, S. (2013b). Representaciones de la escritura académica en contextos de bilingüismo e interculturalidad. Signo y Pensamiento, 32(62), 6480.

Stratilaki, S. (2011). Discours et représentations du plurilinguisme. Frankfurt am Main: Peter Lang.

Universidad-de-Antioquia. (2002). Acuerdo 236 del 30 de octubre de 2002. Medellín.

Velandia, D. (2007). Tutorial Plan to Support the English Speaking Skill of an Inga Student of an Initial Teacher Education Program. Profile, Issues in Teachers' Professional Development, 8, 121-130. 